\title{
Effect of a group-based exercise program on balance in elderly
}

\author{
Tatjana Bulat \\ Stephanie Hart-Hughes \\ Shahbaz Ahmed \\ Pat Quigley \\ Polly Palacios \\ Dennis C Werner \\ Philip Foulis \\ VISN 8 Patient Safety Center, James \\ A. Haley VA Hospital, I 1605 North \\ Nebraska Ave. \\ Tampa, FL 336/2, USA
}

Correspondence:Tatjana Bulat VISN 8 Patient Safety Center, I I 605 North Nebraska Ave. Tampa, FL 336I2, USA

Email tatjana.bulat@va.gov
Objective: To determine the effectiveness of 8-week group functional balance training classes on balance outcomes in community-dwelling veterans at risk for falls.

Design: Pre-test, post-test using retrospective data.

Setting: VISN 8 Patient Safety Center at James A. Haley Veterans Hospital in Tampa, FL, USA.

Participants: Fifty one community living veterans with mean age of 78 at risk for falls. Intervention: Participants received a weekly 1-hour functional balance training class for 8 weeks in a small group setting (4-5 participants).

Measurements: Pre and post intervention measures included Berg Balance Scale, Limits of Stability (LOS) and modified Clinical Test of Sensory Interaction on Balance (mCTSIB).

Results: Eighty four percent of the participants completed 5 or more weekly classes. Peripheral neuropathy was the most common risk factor among the participants. There was a significant improvement in the Berg $(\mathrm{p}<0.0001)$ and Composite Reaction Time $(\mathrm{p}<0.0004)$ after the intervention.

Conclusion: An eight week group functional balance training class was safe and effective in improving balance outcomes in a cohort of elderly veterans at risk for falls.

Keywords: functional balance training, exercise

\section{Introduction}

A growing body of research supports the link between exercise and fall prevention. A systematic review of this literature (Gregg et al 2000), found consistent evidence from a series of prospective and case-control studies linking physical activity with a $20 \%-40 \%$ reduced risk of hip fracture. Balance exercise programs can be effective in improving gait and balance, as well as reducing falls and fall-related injuries (Herwaldt and Pottinger 2003; Wolfson et al 1996; Tinetti et al 1996).

Further research is needed to gain insight into the underlying mechanisms of different type of exercises and their impact on stability in individuals at risk for falls. Despite thousands of research studies published on patient falls, few studies have focused on the effectiveness of interventions (Rubenstein 2004), and fall rates and associated injuries among the elderly continue to rise (CDC 2002). Impaired gait and balance (referred to as impaired stability) is one of the most significant causes and consequences of falls (Robbins et al 1989; American Geriatric Society, British Geriatric Society, and American Academy of Orthopaedic Surgeons Panel on Falls Prevention 2001; Foster et al 2004).

The purpose of this pre-test, post-test clinical intervention study was to determine the effectiveness of 8-week group functional balance training classes on balance outcomes in community-dwelling veterans conducted at the VISN 8 Patient Safety Center from 2001 to 2004 . The objectives of this study were to determine: (1) the diagnostic profile of veterans who participated; (2) class participation rates; (3) the rank order of 
fall risk factors; and (4) differences in balance measures pre and post participation.

\section{Functional balance training}

Functional balance training is a type of exercise that combines muscle strengthening and balance activities with functional gait activities. Regular balance training exercises for a period as short as 9 weeks improved postural control in a study group of persons aged 70-75, as measured by various clinical tests and dynamic posturography, when compared to age-matched control (Ledin et al 1991). A 6-week enhanced balance training program consisting of a series of tasks of increasing difficulty which are related to functional balance versus "standard" physical therapy for adults with balance and mobility deficits (Steadman et al 2003) reported positive results in a sample of 199 older adults. We could find no evidence dealing with the dose or duration, of group balance classes.

\section{Study methods}

\section{Research design}

A pre-test, post test clinical intervention study was conducted over 3 years (2001-2004), using a convenience sample of veterans, that were seen at the VISN 8 Patient Safety Center Falls clinic, found to be at increased risk for falls based on an interdisciplinary fall risk assessment and recommended to participate in an 8-week Group Functional Balance Class.

\section{Clinical intervention}

All class sessions began with the warm-up exercises and were conducted using a standing protocol for each session (see Table 1 describing course content). The specific exercises and patient handouts can be found at http://www.visn8. med.va.gov/patientsafetycenter/fallsTeam.asp The Group Functional Balance Classes incorporated exercises pertaining to lower extremity strengthening, flexibility, coordination, multi-tasking, postural control and gait training. This exercise intervention had a maximum of 5 subjects per class, and was held 1 hour each week, for a total of 8 weeks. The class was taught by a PT with one trained handling assistant for safety reasons. A weekly exercise sheet was given out to participants at the end of each class at which time the therapist demonstrated the home exercises and answered any questions. Participants were provided a course program folder at the beginning of the course, and encouraged to bring the folder to class weekly, so they could take notes, insert new handouts, and show records of their "homework assignments".
Table 1. outlines class content over the 8 week period.

The space accommodated a large set of parallel bars and multiple chairs. Warm-up exercises were performed in a seated position with the participants' chairs being set-up in a "U" shape around the instructor and facilitators. The majority of the standing exercises were performed around the parallel bars (each participant should have ample room to stand and move without touching each other). Chairs were positioned behind each patient to allow them to sit and rest immediately if necessary. Equipment required for each class was listed on each handout. Typically, this equipment consisted of devices which were readily available in a therapy department. Patients were advised to exercise at their "own level" and "listen to their body". They were encouraged to sit (chair located behind them) at any time during the class to rest if necessary and advised to inform a staff member immediately if they experience any form of distress.

The 8 -week curriculum consisted of 8 critical balance elements essential for safe performance of ADLs. These elements progressively increase in difficulty over the 8 week period with increased class time allotted to elements which are more complex and potentially harder for subjects to master. Class begins with an emphasis on core stability exercises in standing and rapidly progresses through various levels of mobility and center of gravity control over the 8 weeks; ultimately ending with multi-tasking and complex movement coordination skills. Protective postural responses, anticipatory postural mechanisms and ability to respond to various environmental conditions (manipulation of surface and vision) are trained throughout the entire 8-week session.

\section{Subjects/sample}

A retrospective chart review of patients that participated in the 8 week Group Functional Balance course were reviewed for the period of 2001-2004. Subject were referred for the course if they were cognitively intact, able to stand unsupported for a minimum of 10 minutes, had fair to good rehab potential demonstrated on Falls Clinic Functional Assessment, were willing to participate and attempt to attend all 8 class sessions, and medically cleared for moderate level exercise. A total of fifty one patients consented to participate in the Group Functional Balance Class.

\section{Measures}

The measures completed pre and post intervention included Berg Balance Scale (Berg et al 1989), Limits of Stability 
Table I Functional balance class content

\begin{tabular}{|c|c|c|}
\hline Week \# & Element trained & $\begin{array}{l}\text { Sample exercises-all exercises performed in } \\
\text { unsupported standing with handhold in close } \\
\text { proximity and close supervision of instructor }\end{array}$ \\
\hline I & Stance stability & $\begin{array}{l}\text { Emphasis initially placed on equal weight bearing. } \\
\text { Participants resist self-initiated and external perturba- } \\
\text { tions (mini squats, "tug of war" with elastic band). } \\
\text { Progressed to eyes closed and compliant surface if } \\
\text { tolerated. }\end{array}$ \\
\hline 2 & Initiation of weight shift & $\begin{array}{l}\text { Weight shifting ant-post/left-right. Ball pass (forced } \\
\text { reaching and weight shift). Looking over shoulder to } \\
\text { promote weight shifting. }\end{array}$ \\
\hline 3 & Advanced weight shifting & $\begin{array}{l}\text { Same as class } 2 \text { yet perform exercises with narrowed } \\
\text { base of support, in step stance position or with lights } \\
\text { dimmed or eyes closed. Add element of timing to tasks: } \\
\text { use metronome or music. }\end{array}$ \\
\hline 4 & Introduction to stepping & $\begin{array}{l}\text { Repeated stepping, alternating side taps or tap-ups to 2" } \\
\text { riser. Kick stationary soccer ball. }\end{array}$ \\
\hline 5 & Dynamic base of support & $\begin{array}{l}\text { Resisted stepping (elastic band around waist and step } \\
\text { away). Multi-directional stepping. Incorporate various } \\
\text { surfaces if capable and/or alter visual input. }\end{array}$ \\
\hline 6 & Vestibular stimulation & $\begin{array}{l}\text { Gaze stabilization exercises in standing. Cone stacking } \\
\text { side to side with associated head motion. }\end{array}$ \\
\hline 7 & Multi-directional locomotion & $\begin{array}{l}\text { Cross-overs, braiding, backwards walking. Direction } \\
\text { changing drills: random, sudden change in directions } \\
\text { requested. }\end{array}$ \\
\hline 8 & High-level coordination activities and multi-tasking & $\begin{array}{l}\text { High-level gait training: speed changes, ambulation with } \\
\text { alternating claps. Dribbling soccer ball, balloon volleyball, } \\
\text { obstacle course }\end{array}$ \\
\hline
\end{tabular}

(LOS) (Clark and Rose 2001) and modified Clinical Test of Sensory Interaction on Balance (mCTSIB) (Shumway-Cook and Horak 1986).

\section{Berg balance scale}

The Berg Balance Scale is a commonly used clinical measure to evaluate performance during various balance activities. The scale consists of 14 common daily balance tasks. Administration requires only minimal basic equipment and takes approximately 15 minutes. All 14 sub-tests are scored on a 5-point ordinal scale based on the subject's ability to perform the requested task safely and in a timely manner. Sub-test scores are summed to achieve a total score ranging from 0 to 56 with higher scores indicating better performance. The Berg Balance Scale has established psychometric properties including a Cronbach alpha value for internal consistency of 0.96 and inter-rater reliability values ranging from 0.71 to 0.99 (Berg et al 1989). Concurrent validity as a measure of balance has been determined in comparison with the POMA balance subscale (Pearson $r=0.91)$ and the Timed Up and Go (Pearson $r=-0.76$ ) (Berg et al 1992).

\section{Limits of stability (LOS)}

This test allows for the analysis of a subject's ability to voluntarily move their center of gravity to their limits of stability in the eight cardinal and diagonal directions. Outcomes include: reaction time, sway velocity, directional control, endpoint excursion and maximum excursion. Endpoint excursion and maximum excursion are calculated as percentages of the subjects theoretical $100 \%$ limit of stability that is a function of their height. The LOS takes 10 minutes to administer and retest reliability has been shown to be good to excellent (Wigglesworth et al 1996).

\section{Modified clinical test of sensory interaction on balance (mCTSIB)}

The mCTSIB protocol consists of four separate conditions, of increasing difficulty, performed with the subject in a quiet upright stance. They are 1) firm surface with eyes open, 2) firm surface with eyes closed, 3) foam surface with eyes open and 4) foam surface with eyes closed. Each sub-test lasts 30 seconds and is repeated 3 times. The combination of these sub-tests can document the presence of sensory dysfunction and provide objec- 
tive analysis of the patient's functional balance control by quantifying postural sway velocity during the four sensory conditions (http://www.onbalance.com/neurocom/ protocols/motorImpairment/los.aspx).

\section{Data collection}

One member of the falls clinic team completed record review. Data were extracted from the Falls Clinic Consult Note (demographics, age, co-morbidities, falls risk factors) and Group Functional Balance Class Notes for each patient (balance outcomes). The data were recorded onto a customized Access database.

\section{Data analysis}

Descriptive statistics and Paired t-test were used for the analysis.

\section{Results}

The age of the participants ranged from 43 to 90 years, with an average age of 77.9 years (SD: 7.2 years) and median age of 80 .

\section{Most commonly identified fall risk factors}

Data were extracted from the fall clinic notes as to the risk factors that were contributing to falls. The most common risk factors were presence of peripheral neuropathy and centrally-acting medications. Since majority of patient has multiple risk factors identified, only top three fall risk factors were extracted from the Falls Clinic Consult Note (Table 2).

\section{Frequency of subject participation}

Forty seven percent of participants attended at least 5 functional balance classes, 22\% attended 7 and 33\% attended all 8 classes.

Table 2 Rank order of fall risk factors

\begin{tabular}{ll}
\hline Risk factor & Number \\
\hline Peripheral neuropathy & 27 \\
Centrally-acting medications & 26 \\
Deconditioning & 19 \\
Pain & 12 \\
Vestibular problems, Dizziness/Vertigo, & $1 \mathrm{I}$ \\
Orthostasis & 9 \\
Residuals of stroke & 8 \\
Impaired Vision & 8 \\
Impaired central sensory integration & 6 \\
Parkinson's disease & 6 \\
Other & 34 \\
\hline
\end{tabular}

Table 3 Berg balance scores

\begin{tabular}{lllll}
\hline Scores & & Mean & SD & p-value \\
\hline Berg score & Pre & 46.80 & 2.85 & $<0.0001$ \\
& Post & 52.50 & 2.73 & \\
\hline
\end{tabular}

\section{Balance outcomes}

Patients made gains in balance in all measures. Increases in scores for post-intervention Berg Balance Scale are presented in Table 3. Mean Berg score increased from 46.8 to 52.5 on average. Participants also showed improvements in Limits of Stability Test (LOS) (See Table 4.) LOS composite Reaction Time (RT) and composite movement velocity (MVL) showed trends toward improvement while LOS composite end point excursion (EPE), maximum excursion (MXE) and directional control (DCL) all were significantly improved when compared to baseline.

Scores on mCTSIB measures also demonstrated improvement represented by a decrease in the mean center of gravity (COG) sway velocity. As shown in Table 5, the lower the number post intervention indicates a trend towards the reduction of sway for all testing conditions. Significant improvement was measured in the composite mean center of gravity sway velocity indicating improvement in balance post intervention (2.50 degrees/sec to 2.20 degrees/sec).

\section{Functional balance scores by frequency of class participation}

A total of 33 participants attended both first and last class. We wanted to explore if there was an optimal dose of the

Table 4 Limits of stability test

\begin{tabular}{|c|c|c|c|}
\hline Variables: & $\begin{array}{l}\text { Pre: } \\
\text { mean(sd) }\end{array}$ & $\begin{array}{l}\text { Post: } \\
\text { mean(sd) }\end{array}$ & p-value \\
\hline LOS_composite RT & $1.19(0.24)$ & $\mathrm{I} .08(0.19)$ & 0.0158 \\
\hline LOS_composite MVL & 2.61 (0.89) & $2.93(0.93)$ & 0.0192 \\
\hline LOS_composite EPE & $40.91(9.19)$ & 49.64 (II.42) & $<0.0001$ \\
\hline LOS_composite MXE & $53.67(10.29)$ & $63.85(13.27)$ & $<0.0001$ \\
\hline LOS_composite DCL & $53.45(10.68)$ & $64.82(10.05)$ & $<0.0001$ \\
\hline \multicolumn{4}{|c|}{$\begin{array}{l}\text { I. Reaction Time (RT) is the time in seconds between the command to move and he } \\
\text { patient's first movement. }\end{array}$} \\
\hline \multicolumn{4}{|c|}{$\begin{array}{l}\text { 2. Movement Velocity (MVL) is the average speed of COG movement in degrees per } \\
\text { second. }\end{array}$} \\
\hline \multicolumn{4}{|c|}{$\begin{array}{l}\text { 3. Endpoint Excursion (EPE) is the distance of the first movement toward the desig- } \\
\text { nated target, expressed as a percentage of maximum LOS distance. The endpoint } \\
\text { is considered to be the point at which the initial movement toward the target } \\
\text { ceases. }\end{array}$} \\
\hline \multicolumn{4}{|c|}{ 4. Maximum Excursion (MXE) is the maximum distance achieved during the trial. } \\
\hline \multicolumn{4}{|c|}{$\begin{array}{l}\text { 5. Directional Control (DCL) is a comparison of the amount of movement in the } \\
\text { intended direction (towards the target) to the amount of extraneous movement } \\
\text { (away from the target). }\end{array}$} \\
\hline
\end{tabular}


Table 5 Modified clinic test for sensory interaction in balance (mCTSIB) results

\begin{tabular}{llll}
\hline Variables: & $\begin{array}{l}\text { Pre: } \\
\text { Mean center of gravity sway } \\
\text { Velocity (degrees/sec)(sd) }\end{array}$ & $\begin{array}{l}\text { Post: } \\
\text { Mean center of gravity sway } \\
\text { Velocity (degrees/sec)(sd) }\end{array}$ & $\begin{array}{l}\text { p-value } \\
\end{array}$ \\
\hline Firm-Eyes Open (firm-EO) & $0.52(0.24)$ & $0.48(0.20)$ & 0.2350 \\
Firm-Eyes Closed (firm-EC) & $0.80(0.65)$ & $0.77(0.40)$ & 0.7648 \\
Foam-Eyes Open (foam-EO) & $3.13(1.83)$ & $2.38(1.68)$ & 0.0039 \\
Foam-Eyes Closed (foam-EC) & $5.55(1.01)$ & $5.18(1.31)$ & 0.0262 \\
Composite & $2.50(0.67)$ & $2.20(0.67)$ & 0.0004 \\
\hline
\end{tabular}

intervention and tried to analyze results by dividing participants based on the number of classes attended (4-6 classes vs. 7-8). Using a paired t-test statistically significant pre-post changes were found in Berg $(\mathrm{p}<.0001)$ and Composite Reaction Time $(\mathrm{p}<.0004)$ for both participants that attended 4-6 and those that attended 7-8 classes when compared to baseline, but none within the groups so, in conclusion, no difference in improvement was found between individuals that participated in 4-6 classes vs. 7-8 classes.

Results are shown in Figure 1.

We also looked at the subgroup of participants with the most common risk factor-peripheral neuropathy and found statistically significant improvement in pre-post measures in Berg score (46.26 [SD 3.03] to 52.74 [SD 2.70]) $(\mathrm{p}<0.0001)$, LOS composite EPE $(\mathrm{p}<0.006)$, composite MXE ( $<<0.001)$, and composite DCL $(\mathrm{p}<0.0005)$ and MCT composite mean COG sway velocity 2.33 (SD 0.78 ) to 1.94 $(\mathrm{SD} 0.66)(\mathrm{p}<0.038)$.

\section{Discussion}

We wanted to provide some preliminary data on dose and intensity of exercise interventions in elderly at risk for falls. We found statistically significant improvement in balance outcomes as a result of an 8 week group functional balance training class. We also demonstrated the feasibility of a group functional balance intervention and safety in elderly at risk for falls. Treating individuals in small groups rather then on a 1:1 basis is also a more economical way of providing treatment. Our study has a number of limitations. It was retrospective and we did not do post-intervention testing on the subjects that dropped out, so we do not know if they were any different then the subjects that finished the intervention. We found improvement (and no difference) in balance outcomes in both patients that participated in 4-6 classes or 7-8 classes since our sample size was probably too small to find a difference. Also we did not measure the amount of time patients spent at home doing exercises on their own which

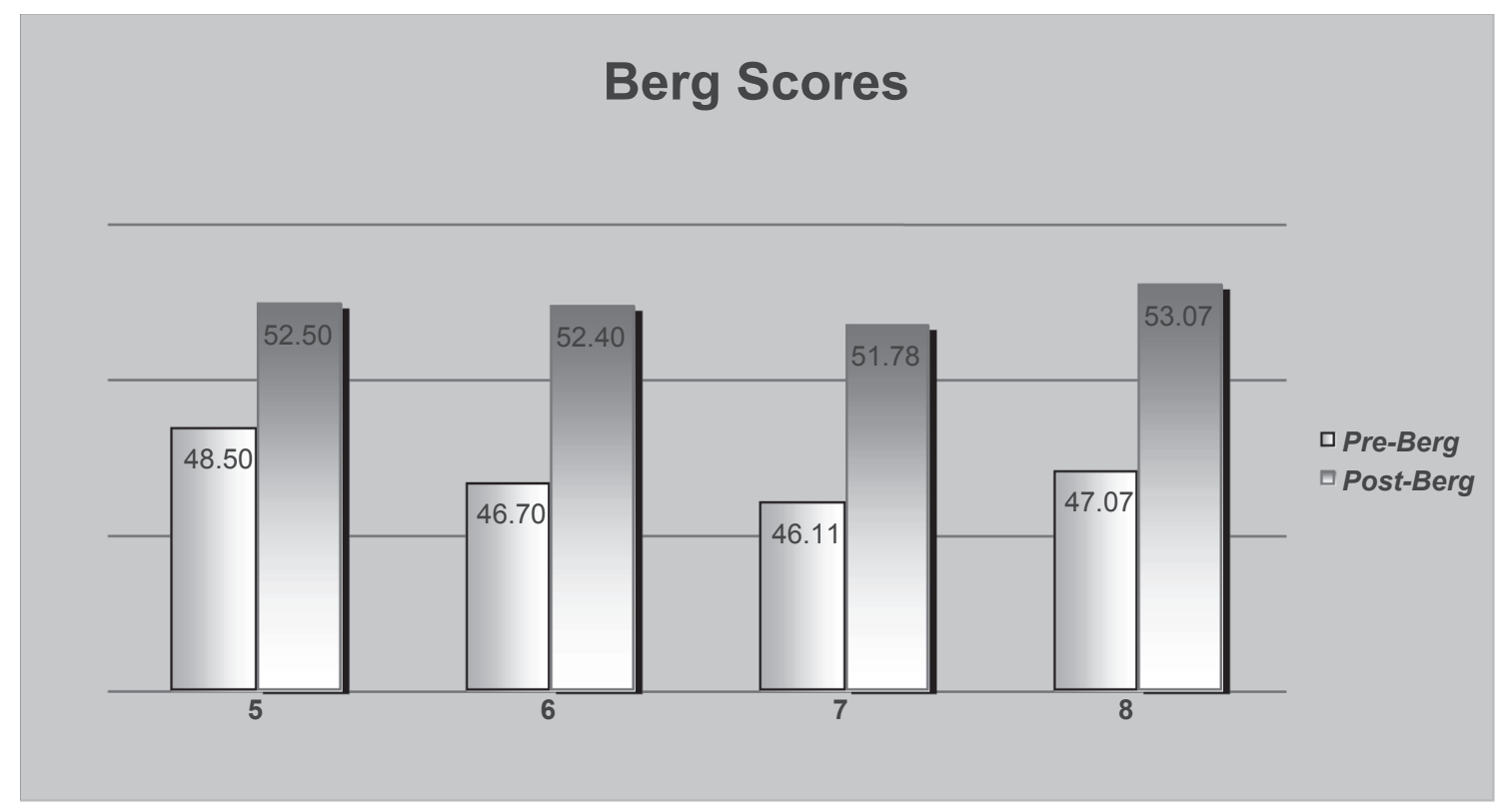

Figure I The distribution of Berg score with respect to differenct classes attended. 
could have confounded the outcomes although that would have been a bigger problem if we did not find the difference in balance measures. We did not track falls but used balance outcomes as a marker of fall risk. In addition, we noticed that our participants benefited form the social interactions (based on their feedback) before, during and after the classes but we did not formally measure their psychosocial wellbeing in this study or how it affected the participation rates.

\section{Future directions}

Our group functional balance intervention needs to be further tested in a prospective trial with falls as a primary outcome rather than balance measures to see if our intervention is effective in reducing fall risk as well as looking at cost-effectiveness of group vs. individual treatment. Further studies are needed to address the most effective dose and intensity of exercise in elderly individuals at risk for falls and testing exercise intervention in specific populations of individuals at risk for falls like peripheral neuropathy, osteoarthritis, Parkinson's disease, etc.

Focusing on a homogeneous patient populations would distinguish unique gait and balance deficits that contribute to impaired stability and mobility risk, as well as to better understand unique responses to treatment that are clouded when diverse patients are aggregated (King and Tinetti 1996; Nelson and Quigley 2002).

\section{References}

American Geriatric Society, British Geriatric Society, and The American Academy of Orthopaedic Surgeons Panel on Falls Prevention. 2001, Guideline For the Prevention of Falls in Older Persons, JAGS, 49, 664-672.

Berg K, Wood-Dauphinee S, Williams JI, Gayton D. 1989. Measuring balance scale: Reliability assessment with an elderly population, Archives of Physical Medicine and Rehabilitation, 73:1073-1080.

Berg KO, Wood-Dauphinee SL, Williams JI, Maki B. 1992. Measuring balance in the elderly: validation of an instrument. Can J Public Health. Jul-Aug, 83 Suppl 2:S7-11.
Berg KO, Wood-Dauphinee SL, Williams JI, Gayton D. 1989. Measuring balance in the elderly: preliminary development of an instrument, Physiotherapy Canada, 41:(304-311.

Centers for Disease Control (CDC). 2002. Injury Fact Book-2001-2002,'US Government Printing Office, Atlanta, GA.

Clark S, Rose DJ. 2001. Evaluation of dynamic balance among communitydwelling older adult fallers: a generalizability study of the limits of stability test, Archives of Physical Medicine and Rehabilitation, 82:468-74.

Foster E, Hillegass LJ, Phillips SL. 2004. Demonstration program: An interdisciplinary approach at a Falls and Mobility Clinic, Annals of Long Term Care, 12 (5):27-32.

Gregg EW, Pereira MA, Caspersen CJ. 2000. Physical activity, falls, and fractures among older adults: A review of the epidemiologic evidence, JAGS, 48:883-93.

Herwaldt L, Pottinger J. 2003. Preventing falls in the elderly, JAGS, vol. $51: 1175-1177$.

King MB, Tinett ME. 1996. A multifactorial approach to reducing injurious falls, Clinics in Geriatric Medicine, 12 (4):745-59.

Ledin T, Kronhead AC, Moller C, Moller M, Odkvist LM, Olsson B. 1991. Effects of balance training in elderly evaluated by clinical tests and dynamic posturography, $J$ Vestib Res, 1 (2):129-38.

Nelson A, Quigley P. 2002. Research Agenda on Risk and Prevention of Falls: 2002-2007, VISN 8 Patient Safety Center of Inquiry, Tampa, FL.

Robbins AS, Rubenstein LZ, Josephson KR, Schulman BL, Osterweil D, Fine G. 1989. Predictors of falls among elderly people: Results of two population based studies, Archives of Internal Medicine, 149: 1628-33.

Rubenstein L. 2004. Joseph T. Freeman Award Lecture. Comprehensive geriatric assessment: from miracle to reality, Journal of Gerontology: Medical Sciences, 59A, (5):473-7.

Shumway-Cook A, Horak, FB. 1986. Assessing the influence of sensory interaction on balance: suggestions from the field, Physical Therapy, 66:1548-50.

Steadman J, Donaldson N, Kalra L. 2003. A randomized controlled trial of an enhanced balance training program to improve mobility and reduce falls in elderly patients, $J A G S, 51$ (6):847-52.

Tinetti ME, McAvay G, Claus E. 1996. Does multiple risk factor reduction explain the reduction in fall rate in the Yale FICSIT trial? American Journal of Epidemiology, 144 (4):389-99.

Wigglesworth JK, Dayhoff NE, Suhrheinrich. 1996. The reliability of four measures of postural control using the Smart Balance Master, $J$ American Coll Sports Med, 29:S113.

Wolfson L, Whipple R, Derby C, Judge J, King M, Amerman P, Schmidt J, Smyers D. 1996, Balance and strength training in older adults: Intervention gains and Tai Chi maintenance, J Am Geriatr Soc, 44: 498-506. 